\title{
Mindfulness
}

\section{A pilot randomized controlled trial for a videoconference-delivered mindfulness-based group intervention in a nonclinical setting --Manuscript Draft--}

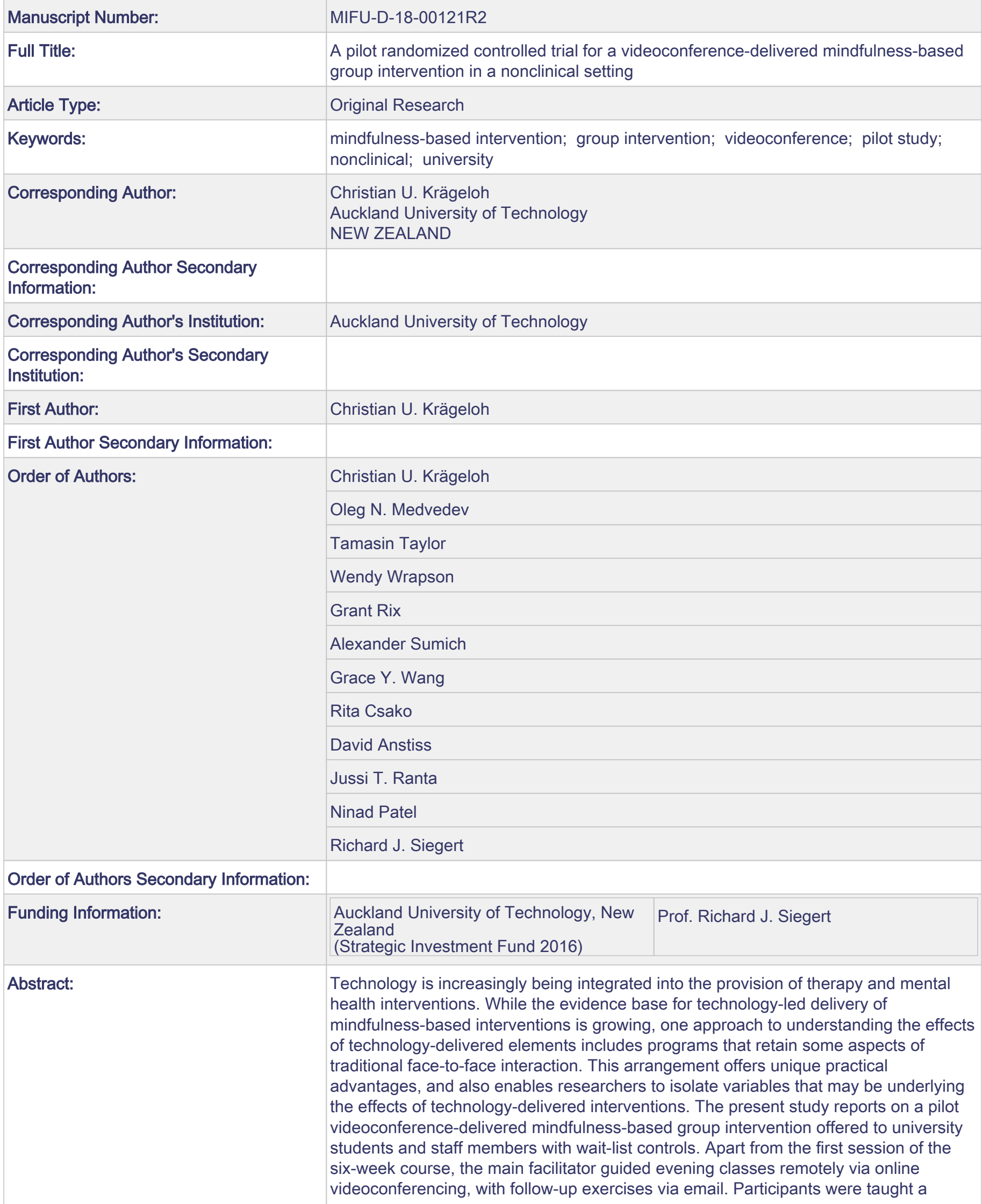




\begin{tabular}{|l|l|}
\hline & $\begin{array}{l}\text { variety of mindfulness-based exercises such as meditation, breathing exercises, } \\
\text { mindful tasting, as well as the concepts underpinning such practice. Participants } \\
\text { completed pre- and post-intervention questionnaires on depression, anxiety, repetitive } \\
\text { negative thinking, dysfunctional attitudes, positive and negative affect, self- } \\
\text { compassion, compassion for others, and mindfulness. For participants who attended at } \\
\text { least five of the six sessions, scores on all outcome measures improved significantly } \\
\text { post intervention and remained stable at three-week follow up. The videoconference- } \\
\text { delivered mindfulness-based group intervention appears to provide a viable alternative } \\
\text { format to standard mindfulness programs where the facilitator and participants need to } \\
\text { live in close physical proximity with each other. }\end{array}$ \\
\hline Response to Reviewers: & $\begin{array}{l}\text { Dear Prof. Singh, } \\
\text { Thank you for the opportunity to submit a revised version of our manuscript. We have } \\
\text { taken the latest feedback on board. After carefully reading through the manuscript a } \\
\text { couple more times, we have also made some additional minor modifications, such as } \\
\text { correcting some minor typographical and grammatical issues. }\end{array}$ \\
& $\begin{array}{l}\text { Best wishes } \\
\text { Chris }\end{array}$ \\
\hline
\end{tabular}




\title{
A pilot randomized controlled trial for a videoconference-delivered mindfulness-based group intervention in a nonclinical setting
}

\author{
Christian U. Krägeloh ${ }^{1 *}$, Oleg N. Medvedev ${ }^{2}$, Tamasin Taylor ${ }^{1}$, Wendy Wrapson ${ }^{1}$, Grant Rix ${ }^{3}$, Alexander \\ Sumich ${ }^{1,4}$, Grace Y. Wang ${ }^{1}$, Rita Csako ${ }^{1}$, David Anstiss ${ }^{1}$, Jussi T. Ranta ${ }^{1}$, Ninad Patel ${ }^{1}, \&$ Richard J. Siegert $^{1}$ \\ ${ }^{1}$ Auckland University of Technology, New Zealand \\ ${ }^{2}$ University of Auckland, New Zealand \\ ${ }^{3}$ Mindfulness Education Group, New Zealand \\ ${ }^{4}$ Nottingham Trent University, United Kingdom
}

${ }^{*}$ Corresponding author: Chris Krägeloh, School of Public Health and Psychosocial Studies, Faculty of Health and Environmental Sciences, Auckland University of Technology, 90 Akoranga Drive, Auckland 1142, New Zealand; email: chris.krageloh@aut.ac.nz

Running head: Videoconference-delivered mindfulness-based group intervention

ORCID numbers of authors:

Christian U. Krägeloh: 0000-0002-7298-0736

Oleg N. Medvedev: 0000-0002-2167-5002

Grace Y. Wang: 0000-0003-2063-031X

\begin{abstract}
Technology is increasingly being integrated into the provision of therapy and mental health interventions. While the evidence base for technology-led delivery of mindfulness-based interventions is growing, one approach to understanding the effects of technology-delivered elements includes programs that retain some aspects of traditional face-to-face interaction. This arrangement offers unique practical advantages, and also enables researchers to isolate variables that may be underlying the effects of technology-delivered interventions. The present study reports on a pilot videoconference-delivered mindfulness-based group intervention offered to university students and staff members with wait-list controls. Apart from the first session of the six-week course, the main facilitator guided evening classes remotely via online videoconferencing, with follow-up exercises via email. Participants were taught a variety of mindfulnessbased exercises such as meditation, breathing exercises, mindful tasting, as well as the concepts underpinning such practice. Participants completed pre- and post-intervention questionnaires on depression, anxiety, repetitive negative thinking, dysfunctional attitudes, positive and negative affect, self-compassion, compassion for others, and mindfulness. For participants who attended at least five of the six sessions, scores on all outcome measures improved significantly post intervention and remained stable at three-week follow up. The videoconference-delivered mindfulness-based group intervention appears to provide a viable alternative format to standard mindfulness programs where the facilitator and participants need to live in close physical proximity with each other.
\end{abstract}

Key words: mindfulness-based intervention; group intervention; videoconference; pilot study; nonclinical; university;

Authors' Contributions: RS led the funding application for this project. AS, CK, GW, RS, and WW planned and designed the study. The mindfulness intervention was delivered by GR, with assistance of RC, TT, JR, and CK. TT coordinated recruitment and data collection. DA, JR and OM assisted in the coordination of the study. Data were analyzed by OM, TT, CK, and NP. The majority of the write up was completed by CK, with help by OM and NP. All authors contributed to the manuscript and approved the final version.

Conflict of Interest: GR provides mindfulness-based interventions in educational settings as part of a business. These interventions currently do not follow the videoconference-delivered approach reported here, but may in the future. 


\title{
A pilot randomized controlled trial for a videoconference-delivered mindfulness-based group intervention in a nonclinical setting
}

\begin{abstract}
Technology is increasingly being integrated into the provision of therapy and mental health interventions. While the evidence base for technology-led delivery of mindfulness-based interventions is growing, one approach to understanding the effects of technology-delivered elements includes programs that retain some aspects of traditional face-to-face interaction. This arrangement offers unique practical advantages, and also enables researchers to isolate variables that may be underlying the effects of technology-delivered interventions. The present study reports on a pilot videoconference-delivered mindfulness-based group intervention offered to university students and staff members with wait-list controls. Apart from the first session of the six-week course, the main facilitator guided evening classes remotely via online videoconferencing, with follow-up exercises via email. Participants were taught a variety of mindfulness-based exercises such as meditation, breathing exercises, mindful tasting, as well as the concepts underpinning such practice. Participants completed pre- and post-intervention questionnaires on depression, anxiety, repetitive negative thinking, dysfunctional attitudes, positive and negative affect, self-compassion, compassion for others, and mindfulness. For participants who attended at least five of the six sessions, scores on all outcome measures improved significantly post intervention and remained stable at three-week follow up. The videoconference-delivered mindfulness-based group intervention appears to provide a viable alternative format to standard mindfulness programs where the facilitator and participants need to live in close physical proximity with each other.
\end{abstract}

Key words: mindfulness-based intervention; group intervention; videoconference; pilot study; nonclinical; university; 


\section{Introduction}

technology has increasingly been applied to deliver mindfulness interventions or support mindfulness practice. This includes a variety of approaches, ranging from phone-delivered mindfulness training sessions (Salmoirago-Blotcher et al. 2012) and mindfulness-based mobile applications (Plaza et al. 2013; van Emmerick et al. 2018), to mindfulness in self-help interventions delivered through websites (Gu et al. 2018) or mindfulness taught using a combination of a virtual online classroom and website (Aikens et al. 2014).

A recent meta-analysis by Spijkerman et al. (2016) examined the effectiveness of 15 online mindfulness-based interventions (MBIs) to improve mental health. Eight of the fifteen studies delivered a mindfulness-based stress reduction (MBSR; Kabat-Zinn 1990) program, two mindfulnessbased cognitive therapy (MBCT; Williams et al. 2007), and five an acceptance and commitment therapy (ACT; Hayes et al. 1999) intervention. Guidance from a therapist was given during the interventions in nine studies, and in five of these, participants were given feedback and individual coaching (e.g. positive encouragement, answering questions) through email, telephone or encrypted webpages. In three studies, guidance was given weekly in 1- or 2-hour online classes (group-based), with one also providing supplementary (pre-programmed) individual email coaching. MBIs were predominantly delivered through websites $(n=14)$. Further delivery modes included a smartphone application $(n=1)$ and also a virtual online classroom $(n=2)$. Intervention durations varied from 2 to 12 weeks, and the sessions were usually conducted weekly. Overall, the meta-analysis found small but significant effect sizes for anxiety, depression, stress, well-being, and mindfulness (Spijkerman et al. 2016). Although previous research has demonstrated that online psychological interventions are equivalent to traditional face-to-face interventions in terms of effectiveness (Barak et al. 2008), the effect sizes observed in this meta-analysis were found to be generally lower than those of face-to-face 
MBIs (Abbott et al. 2014; Cavanagh et al. 2014; Gotink et al. 2015). This may suggest that, as of yet,

While mechanisms underlying the effectiveness of online MBIs are still being proposed and tested (Cavanagh et al. 2018), another avenue of research is through so-named blended web-based mindfulness programs. Montero-Marin et al. (2018) combined traditional face-to-face interaction with online-based practice sessions. During an initial four-hour face-to-face group session, general medical practitioners were introduced to the theoretical background of mindfulness, its usefulness for their professional practice, and how to implement this practice into their daily lives. The program was modelled on MBSR, but was designed to be brief and thus did not contain a full-day workshop as is typical for MBSR. Subsequent to this face-to-face session, participants were provided with four 45min sessions of online training. These were to be completed within a period of two weeks and presented participants with audio, video, and text material for guided practice and further theoretical description. However, during this online phase, participants received no supervision or feedback from the instructor or any of the researchers. Only 30 of the 290 participants completed two or more of the weekly practices, and, for those participants, significant improvement in positive affect (moderate effect size) and mindfulness (small effect size) was noted. There were no significant effects on secondary outcome measures such as negative affect, resilience, or burnout.

A limitation of the study by Montero-Marin et al. (2018) was the low practice adherence, which may be expected for health professionals such as general practitioners who are frequently reported to have a very high workload (Thompson and Walter 2016). However, the blended approach of combining face-to-face and online delivery of mindfulness intervention has the potential to offer unique advantages and thus warrants further investigation and development. Compared to purely online-based MBIs, blended programs will not pose such a stark contrast to conventional face-to-face delivery facilitating the acceptability of such interventions for participants who may not be particularly comfortable with fully online group programs. Blended programs may thus be perceived as a compromise between the two extremes. For others who are happy with online formats, some may 
additionally appreciate having met the facilitator in person before continuing with the program online. are manipulated when introducing online elements into delivery of MBIs, which may help isolate variables that are associated with treatment effectiveness.

A different type of blended approach was taken by Magtibay et al. (2017) - in this case blended learning, where participants were able to choose the format that met their learning styles and goals. Fifty nurses self-selected to participate in an intervention for mindfulness and resilience to address stress and burnout, which had been identified as common issues in this target population. Depending on personal preference, participants could choose between various options for learning the content: online-based formats, independent reading, facilitation, or a combination of those. There was no requirement to complete the exercises within a specified time period, although participants were encouraged to complete the online-based portion within eight weeks. At Weeks 8 and 12, sessions were conducted face to face, and by telephone during Weeks 16 and 20. During some of these weeks and at follow up, participants also completed a number of questionnaires to assess stress, burnout, happiness, and mindfulness. While attendance as these follow-up sessions was poor $(20,15,2$, and 1 in Weeks $8,12,16$, and 20 , respectively), completion rates for the survey were substantially higher (50, 45, 40, and 33 for baseline, Weeks 8, 12, and 24, respectively). Each outcome measure changed at each assessment point in the expected direction. Although effect sizes were not presented, these can be calculated (comparing baseline with Week 24) from the data provided: small effect size for resilience (Cohen's $d=0.43)$; medium effect sizes for happiness $(d=0.61)$, client-related burnout $(d=0.68)$, and perceived stress $(d=0.74)$; and large effect sizes for mindfulness $(d=0.80)$, generalized anxiety disorder $(d=0.87)$, work-related burnout $(d=0.88)$, and personal burnout $(d=0.89)$.

The present article reports on a pilot study of a videoconference-delivered mindfulness-based group intervention - thus a blend between a traditional group format and a technology-delivered program. This program was delivered in a university setting as it likely ensures higher treatment adherence than in previous studies of blended approaches (Magtibay et al. 2017; Montero-Marin et al. 
2018). Additionally, university environments are known to be demanding and stressful where maintaining well-being and a healthy lifestyle can be challenging for both staff and students, making this a population that could benefit particularly well from MBIs (Henning et al. 2018). Unlike the study by Montero-Marin et al. (2018), the mindfulness program delivered in the present study retained the group format of standard MBIs such as MBSR and MBCT. The program reported here thus provides an approach to investigating the effects of specific aspects of technology-facilitated content delivery as opposed to offering an experience that differs from standard MBI on a number of dimensions. In our case, the technology-facilitated element was limited to the facilitator who delivered the group MBI remotely from the second session onward. The study participants thus met the facilitator in person during the first session, which allowed them to build initial rapport.

The primary purpose of this pilot study was to test the feasibility of a videoconference approach to delivering a mindfulness-based group intervention, which included gaining information about recruitment and treatment adherence. Prior power calculation is not necessarily a feature of such pilot work (Arain et al. 2010; Thabane et al. 2010), although the purpose of the study was to explore expected effect sizes for various outcome measures of interest. As this videoconference-based mindfulness program was delivered in a group format, it was expected that effect sizes may be comparable to the moderate effect sizes found for group MBIs in nonclinical settings (Khoury et al. 2015). It was thus hypothesized that the participants will exhibit post-intervention increases in outcome measures related to psychological well-being, such depression, anxiety, dysfunctional attitudes, or positive and negative affect.

\section{Method}

\section{Participants}

Participants were individuals either studying or working at Auckland University of Technology, New Zealand. The study was advertised to students and staff through posters, emails 
delivered through various university communication channels, Facebook pages specifically set up for the study, and presentations to students at the beginning of their classes. A total of 204 individuals expressed their interest in participating in the research, of which three did not meet the inclusion criteria of absence of psychological condition requiring ongoing medication, absence of epilepsy or brain injury, and no alcohol of substance abuse. The remaining 201 interested individuals were randomly allocated to either Group 1 or Group 2 (waitlist control group with delayed start) and then contacted via email and/or telephone to confirm their participation. Of those, 42 initially confirmed their participation in the study, but a further ten were not able to continue. The number of participants attending at least one session was 15 for Group 1 and 17 for Group 2. Figure 1 shows an overview of the participant allocation outlined above.

\section{〈PLEASE INSERT FIGURE 1 ABOUT HERE〉}

Of these 32 participants, 24 were undergraduate students, 6 were enrolled in post-graduate studies, and 2 were staff. Eleven identified as male (34\%) and 21 as female (66\%). The mean age was 30.06 years, with a standard deviation of 10.94 and a range of 18 to 58 years. The ethnic makeup was diverse, including New Zealand European ( $n=14 ; 44 \%)$, Asian ( $n=6 ; 19 \%)$, Indian $(n=3 ; 9 \%)$, Māori $(n=1 ; 3 \%)$, Pacific Islander $(n=1 ; 3 \%)$, and others or not specified $(n=7 ; 22 \%)$. There were no notable differences in terms of demographic profile across Groups 1 (immediate start) and 2 (wait list control with delayed start).

\section{Procedure}

Mindfulness sessions took place in a quiet classroom between 17:00 to 19:00 hours. At all times, a clinical psychologist was present who also participated in the exercises but who identified herself as staff who was able to help if any of the students were to experience any psychological distress. Typically, one additional researcher was also present who helped set up the room and tidied up afterwards and also made sure that the videoconferencing technology was working. 


\section{<PLEASE INSERT TABLE 1 ABOUT HERE>}

From Session 2, the facilitator guided the sessions via videoconferencing using GTM. The second session comprised of a 10 to 15 -minute breathing meditation exercise, a physical movement exercise similar to Taijiquan, and a slideshow presentation on mindfulness and the brain. The session concluded with a brief guided meditation exercise and a mindful tasting exercise. Session 3 also 
included the physical exercise as well as guided breathing meditation. A slideshow presentation was given by the main facilitator on types of awareness, negative bias, and advantages of walking meditation. Session 4 included physical breathing exercises, breathing meditation, and a 45-minute talk on the foundations of mindfulness and emotion. The session similarly concluded with a meditation exercise, which focused on observing sound, body, and emotions. Session 5 contained the physical movement exercise, concentration meditation, and a talk on accepting and regulating emotion. Session 6 involved physical movement and a breathing exercise, meditation practice, as well as a discussion on the four foundations of mindfulness and its purpose. The facilitator also discussed loving kindness meditation, and explained it as involving repeating mantras to mentally send warmth, goodwill, and kindness to others.

At various stages during the face-to-face session and the sessions delivered via videoconferencing, the facilitator encouraged interaction with and among the participants. This could be in the form of a discussion but also as brief feedback. For example, immediately after the guided meditation session in Session 3, participants were asked to describe with one word how they felt. All sessions concluded with questions and answers.

Group 1 started the six-week mindfulness program three weeks prior to Group 2. As there was a three-week overlap period, the groups met on different days of the week. The sessions in Group 2 followed the same outline. However, the program occasionally had some minor variations, in response to questions by participants such as requests to elaborate on certain material or exercises. Due to logistical reasons, the mindful tasting exercise was conducted for Group 2 during Session 3 and not Session 2. Any other differences in session content were minimal. Apart from technical problems occurring in one of the sessions, which delayed the start by 15 minutes, there were no noteworthy issues with the equipment.

Participants were encouraged to practice for at least 15 minutes per day. Exercises to be practiced were either those covered in class or those shown by following a link to audio or video files 
sent to the participants after each session. A day before the start of the next session, participants were sent a reminder email as well as a link to an online questionnaire inquiring about their home practice during that week. This included questions about frequency of practice during the week and average length of practice.

For both groups, baseline measures were taken during the week immediately prior to commencement of the mindfulness program. As Group 2 started the mindfulness program three weeks after Group 1, there was an opportunity to collect baseline measures twice, which served as a means to control for history effects as well as learning effects from repeated assessment. For both groups, a post-intervention measure was obtained during the first week following the final session of the mindfulness program. As Group 1 finished their program three weeks prior to Group 2, a second postintervention measure could be obtained for Group 1, which served as a three-week follow-up measure. The timeline is illustrated in Table 2. Out of 15 participants in Group 1 who completed at least one mindfulness sessions, 13 completed the questionnaires. Twelve participants in Group 1 completed the first post-intervention measure, of which eight participants had completed at least five of the total of six sessions. For Group 2, the number of participants who completed at least five sessions was also eight.

Ethical approval was obtained from the authors' institutional ethics committee, and written informed consent was given by all participants. This study was part of a larger trial that investigated the effects of mindfulness on brain function and biomarkers. Some baseline data relating to biomarkers have already been published elsewhere (Wang et al. 2017).

<PLEASE INSERT TABLE 2 ABOUT HERE>

\section{Measures}


As part of the larger study investigating the relationship between self-report measures of mindfulness, depressive symptoms, anxiety, compassion, and self-compassion with brain function and biomarkers, participants made appointments with one of the researchers to complete a variety of tests. This was scheduled according to the availability of the participants as it included several other behavioral and physiological assessments. The following psychometric instruments were completed online using the software Qualtrics. Respondents were required to answer all items in each questionnaire.

Depression. Participants completed the Beck Depression Inventory II (BDI; Beck et al. 1961, 1996). This questionnaire has 21 items where each item presents four statements from which the respondent needs to select one. The four statements are scored $0,1,2$, or 3, and a higher score reflects a greater degree of depression. For example, one item lists the word "Sadness" followed by the options "I do not feel sad", "I feel sad much of the time", "I am sad all of the time", and "I am so sad or unhappy that I can't stand it". The BDI-II has been used with samples including university students (de Sá Junior et al. 2018), and there is also good psychometric evidence for the use of a single general score (de Sá Junior et al. 2018; Siegert et al. 2009), which was used in the present study.

Anxiety. The Beck Anxiety Inventory (BAI; Beck et al. 1988) presented 21 items that describe symptoms of anxiety (e.g., "Numbness or tingling"). Respondents rate each item on a fourpoint Likert scale ranging from "Not at all" to "Severely (I could barely stand it)", which are added to a summary score, where higher scores represent higher levels of anxiety. The BAI has been used with a variety of populations including university students, with good psychometric properties including Cronbach's alpha above .90 (Julian 2011).

Dysfunctional attitudes. Dysfunctional attitudes linked to vulnerability to depression were measured using one of the short forms of the Dysfunctional Attitudes Scale (DAS-SF $F_{1}$; Beevers et al. 2007). The nine items of this scale (e.g., "If I don't set the highest standard for myself, I am likely to 
end up a second-rate person.") were presented using a four-point Likert scale (ranging from "Totally agree" to "Totally disagree"). Individual item scores were summed together to yield a summary score where a higher score indicates a lower level of dysfunctional attitudes. The scale was originally developed using university student samples and shows good psychometric properties including Cronbach's alpha above .80 (Beevers et al. 2007).

Repetitive negative thinking. The Perseverative Thinking Questionnaire (PTQ; Ehring et al. 2011) presents 15 items (e.g., "The same thoughts keep going through my mind again and again.”) on a five-point Likert scale ranging from "Never" to "Almost always". Items are summed together to a total score, where a higher score indicates a higher level of repetitive negative thinking. The scale was originally developed and validated with samples that included university students, with good psychometric properties such as Cronbach's alpha above .90 (Ehring et al. 2011).

Positive and Negative affect. The 20-item Positive and Negative Affect Schedule (PANAS; Watson et al. 1988) lists 20 adjectives (e.g., "Interested", "Distressed", "Excited", and "Upset") and asks the participant to indicate on a five-point Likert scale (ranging from "Very slightly or not at all" to "Extremely") the extent to which each adjective indicates how much they currently feel this way. The scores of ten items are summed to generate a total score of positive affect (PA), and the remaining ten indicate level of negative affect (NA). The original development and validation work of the scale included samples of university students, which demonstration good psychometric properties such as Cronbach's alpha above .80 (Watson et al. 1988).

Self-compassion. The 12-item short form of the self-compassion scale (Raes et al. 2011) was used to measure self-compassion. Unlike the full-length self-compassion scale (Neff 2003), which contains six sub-scales, the short form produces a single score of self-compassion. The short form presents two items from each of the six sub-scales using a five-point Likert format ("Almost never" to "Almost always"). An example item is "When I fail at something important to me, I become consumed by feelings of inadequacy" (over-identification sub-scale). Items from the sub-scales 
isolation, over-identification, and self-judgment are reverse coded before adding all items together to form has been validated in samples with university students, with Cronbach's alpha exceeding .80 (Raes et al. 2011).

Compassion. The compassion scale (Pommier 2010) contains 24 items presented in a fivepoint Likert scale format ("Almost never" to "Almost always"). Items are grouped into six sub-scales of four items each. An example item is "When people cry in front of me, I often don't feel anything at all” (disengagement sub-scale). After reverse coding items from three of the sub-scales (disengagement, indifference, and separation), a total score can be calculated by summing all items so that a higher score represents a higher level of compassion. The original validation work of the scale included university student samples, with Cronbach's alpha for the total score reported as exceeding .80 (Pommier 2010).

Mindfulness. The 39-item Five Facet Mindfulness Questionnaire (FFMQ; Baer et al. 2006) assesses aspects of mindfulness grouped into one of five sub-scales: Act (e.g., "I am easily distracted"), Describe (e.g., "I am good at describing the words to describe my feelings"), Nonjudge (e.g., "I criticize myself for having irrational or inappropriate emotions"), Nonreact (e.g., "I perceive my feelings and emotions without having to react to them"), and Observe (e.g., "When I am walking, I deliberately notice the sensations of my body moving"). Questions are scored on a five-point Likert scale ranging from "Never or very rarely" to "Very often or always true". After recoding negatively worded items, higher scores indicate a higher level of mindfulness. Data were converted from ordinalto interval-level scores for each sub-scale according to the algorithms recommended by Medvedev et al. (2017). These conversion algorithms were based on validation work that included university student samples. Reliability of this scale was measured using person separation index (PSI), which is interpreted in a similar way to Cronbach's alpha (Tennant and Conaghan 2007). Reliability was acceptable, with PSI values of the five sub-scales ranging from .76 to .89 . 


\section{Data analyses}

Analyses were conducted using the software SPSS v25. Given the small sample size, nonparametric tests were conducted, namely paired-samples Wilcoxon rank-sum test for within-group comparisons (such as pre- versus post-intervention scores) and Mann-Whitney U test for betweengroup comparisons such as when comparing baseline scores of Groups 1 and 2. Analyses of this pilot study explored patterns of change such as movement of all measures in expected directions. A nonparametric sign test was conducted to test whether movement in scores was significantly in one direction (Siegel and Castellan 1988). Therefore, if the overall movement of scores in the expected direction was significant according to this sign test, it can be concluded that this change was unlikely due to inflation of Type-1 error rate.

\section{Results}

The baseline scores of Groups 1 and 2 were comparable for all measures. Even though there were 15 participants in Group 1 who took part in at least one of the mindfulness sessions, only 13 of those completed the Pre1 questionnaires immediately prior to the intervention (Table 2). In contrast, all participants in Group 2 completed the Pre1 questionnaires. Comparing the Pre1 scores across groups, the only significant differences were for NA (Group 1 mean=25.00, SD=8.24, Group 2 mean=19.31, $\mathrm{SD}=7.89, U=56.50, p<.05$ ) and the Nonjudge sub-scale of the FFMQ (Group 1 mean=23.21, $\mathrm{SD}=5.83$, Group 2 mean=26.80, $\mathrm{SD}=3.75, U=41.00, p<.01)$. When comparing the Pre0 scores of Group 2 with the Pre 1 scores of Group 1 (both of which were collected during the same week), only the scores for Nonjudge were significantly different $(U=60.50, p<.05)$. At Pre 0 , the mean Nonjudge score for Group 2 was 25.02 ( $\mathrm{SD}=2.58$ ). The comparisons of Pre0 scores with Pre1 scores indicated that the baseline scores for Group 2 remained stable. The only significant difference 
between these two time points was noted for BDI $(z=-2.67, p<.01)$, which increased from 7.19 $(\mathrm{SD}=6.18)$ to $10.12(\mathrm{SD}=7.32)$.

Table 3 shows attendance at each mindfulness session of Groups 1 and 2. Attendance dropped steadily throughout the six-week course. The lowest number of participants was in Sessions 4 and 5, but attendance increased again for the final session. Four of the initial 32 participants attended only one $(n=1)$ or two sessions $(n=3)$ and were not available for post-intervention assessment. Of the remaining 28 participants, six participants each attended three and four sessions, while eight participants each attended either five or six sessions. Of the 12 participants who came to either three or four sessions, seven provided Pre1 and Post 1 data, and for the 16 participants who attended either five or six sessions, Pre1 and Post1 data were available for 15 participants.

\section{<PLEASE INSERT TABLE 3 ABOUT HERE >}

The pre- and post-intervention results for all measures are shown in Table 4. Results are presented separately for the entire sample of 22 participants for whom pre- and post-interventions scores were available $(n=22)$, those participants who only attended three or four of the six sessions $(n=7)$, and those who attended either five or six sessions $(n=15)$. However, the pre-post comparisons did not reach statistical significance for those who only attended three out of four sessions. While the effect sizes were clearly smaller for this sub-group, the lack of statistical significance is very likely also due to the small sample size.

\section{<PLEASE INSERT TABLE 4 ABOUT HERE >}

For the participants who completed five or six sessions, scores changed significantly for all scales except for positive affect (PA), compassion for others (CS), FFMQ Describe, and FFMQ Observe. All significant changes were in the expected directions, with negative change for depression (BDI), anxiety (BAI), repetitive negative thinking (PTQ), negative affect (NA) and positive change 
for self-compassion (SCS), total mindfulness score (FFMQ total), acting with awareness (FFMQ Act), nonjudging (FFMQ Nonjudge), and nonreactivity (FFMQ Nonreact). Also note that the DAS scores increased significantly, and here a higher score indicates lower levels of dysfunctional attitudes. Effect sizes for these changes ranged from small (Cohen's $d \geq 0.20$ ) for BAI, DAS, PTQ, and FFMQ Nonreact, medium (Cohen's $d \geq 0.50$ ) for NA and FFMQ Nonjudge, to large (Cohen's $d \geq 0.80$ ) for BDI, SCS, and FFMQ total. Not considering the FFMQ total scores as they are not independent of the sub-scale scores, all of the nine significant changes in outcomes measures were in expected directions (BDI, BAI, DAS, PTQ, NA, SCS, FFMQ Act, FFMQ Nonjudge, and FFMQ Nonreact). According to a sign test, a movement of nine out of nine scores in one direction is statistically significant (twotailed, $p<.02)$.

The second post-intervention data collection (Post2) for Group 1 allows an assessment of post-intervention follow-up. Comparing the Post 1 scores with Post 2 scores of the participants who completed at least five mindfulness sessions ( $n=7 ; n=6$ for DAS), only two significant differences were noted: The decrease in the PTQ mean from Post1 $(\mathrm{M}=30.29, \mathrm{SD}=7.74)$ to Post2 $(\mathrm{M}=26.71$, $\mathrm{SD}=10.14)$ was significant $(z=-2.00, p<.05)$ as well as the increase in FFMQ Nonreact $(z=-2.00$, $p<.05)$ from $16.43(\mathrm{SD}=2.87)$ to $17.88(\mathrm{SD}=2.95)$.

Data on home practice was relatively incomplete as the overall response rate to questions about home practice was $58 \%$. However, of those 15 participants who completed at least five sessions, 14 participants provided data for at least four of the five times they were asked. For those 14 participants, the average frequency of practice and average length of practice was calculated for the six-day period since the last mindfulness session. Excluding one outlier who indicated practicing 50.00 min per day, the median frequency was 2.50 and median length of daily practice time was 14.00 min (range 5.00 to $18.80 \mathrm{~min}$ ). Average frequency of practice was not correlated (Spearman's rho) with change scores of any of the outcome measures. For length of practice per day, significant correlations were found for only two of the outcomes measures, and in both cases in unexpected directions: Pre1-to-Post1 change scores of the FFMQ Describe were negatively correlated (rho=-.58, 
$p<.05)$ with practice length, and also for FFMQ Nonreact (rho=-.59, $p<.05$ ). However, given the small

\section{Discussion}

The present study reported on a pilot videoconference-delivered group MBI in a nonclinical setting. For participants who attended at least five of the six sessions of the mindfulness program, significant reductions of depression, anxiety, repetitive negative thinking, negative affect and dysfunctional attitudes and significant increases of self-compassion, total mindfulness, acting with awareness, nonjudging, and nonreactivity were evident. The changes were particularly large for depression, self-compassion, and overall mindfulness, as indicated by effects sizes above 0.80 . Overall, this approach of delivering a mindfulness intervention program was effective and brought about positive change in participants that is higher than those reported in online-based MBIs (Spijkerman et al. 2016) and more comparable to those found in face-to-face group MBIs in nonclinical settings (Khoury et al. 2015). The limited evidence from the follow-up data indicated that the effects remained for at least three weeks after the intervention.

The similarity of the results of the present study with those of traditional face-to-face group MBIs rather than online formats might be related to the fact that the facilitator in our study was present during the first session, which allowed participants to build rapport. When introducing himself to the group, the facilitator provided background about his own personal mindfulness practice, thus potentially establishing himself as a role model for course participants. The importance of so-called embodiment of mindfulness practice by the teacher has increasingly been recognized in the literature as an important variable that could affect the effectiveness of an MBI (Broderick et al. 2018). Future studies might deliberately arrange different conditions that enhance or diminish such embodiment as perceived by the participants. 
In the present study, no changes were found for positive affect, FFMQ Observe, and FFMQ Describe. The lack of an effect of the present intervention on the Observe sub-scale of the FFMQ may not be entirely surprising given the fact that it has frequently been reported to present with unexpected relationships with variables of psychological well-being (Rudkin et al. 2018). Similarly, the FFMQ Describe sub-scale has been found to yield occasional contradictory associations (Fernandez et al. 2010) and has also been described as theoretically problematic (Christopher et al. 2014; Feng et al. 2018). The lack of an effect on compassion for others may be a reflection of the focus of the present program more on personal observations and self-compassion, although some loving kindness practice had been incorporated. While the link between mindfulness and compassion has been argued conceptually (Krägeloh 2016), empirical evidence suggests that a fair amount of explicit practice focusing on compassion for others is required to develop this characteristic (Brito-Pons et al. 2018).

Out of the initial 32 participants, only 23 remained during the final session of the six-week program, and only 15 participants attended at least five sessions. This attrition rate is not dissimilar to those found in eTherapy. Richards and Richardson (2012) published a meta-analysis of 40 studies on computer-based psychological interventions. The dropout rate for unsupported treatments was almost $75 \%$, compared to $38 \%$ for those with administrative support, and $28 \%$ for those with therapist support. Even values for conventional face-to-face psychological therapy range from 30 to $60 \%$ (Richards and Richardson 2012). Forbes et al. (2018) provided a detailed analysis of adherence rates for an online-based MBI for university students. After the first meditation exercise, $16.5 \%$ of participants dropped out, followed by a steady decline to around $50 \%$ after the $10^{\text {th }}$ daily meditation. Other reports of attrition rates include ranges from $8 \%$ to more than $50 \%$ for MBIs delivered via technology with no facilitator involvement (Fish et al. 2016).

Poor adherence will likely decrease the effectiveness of the intervention, which may be particularly the case in mindfulness training, as regular practice is considered essential in acquiring mindfulness skills (Lacaille et al. 2017; Spijkerman et al. 2016). Because the studies in the meta- 
analysis by Spijkerman et al. (2016) used diverse definitions of adherence and often lacked clarification of how adherence was measured, the authors did not rule out that non-optimal adherence rates may have prevented the full potential of online MBIs. This poses the question of how adherence can be enhanced in online MBIs. Prior research has suggested that providing support has a positive influence on adherence and also enhances the effectiveness of online interventions (Andersson and Cuijpers 2009). On the other hand, there may be aspects of the delivery format that cannot be easily addressed in program designs as participants may have particular preference due to a variety of reasons. As reported by Lauricella (2014), for example, about half of the university students in their sample preferred a face-to-face mindfulness exercise, while a quarter preferred digital practice. As individuals gain more familiarity with online formats, their preference may either shift more in favor of these approaches, or individuals may self-select for these types of individuals with more realistic expectations. Increasing adherence is an important goal as it is often individuals with higher baseline levels of depressive rumination that tend to drop out of MBIs (Banerjee et al. 2018), and these individuals are particularly in need of this intervention.

The results of the present pilot study provide useful information about the extent of recruitment activities that might be required for a future full trial. In response to the first wave of advertisement for this pilot study, 201 individuals registered interest in the program. Of those, 42 confirmed their continued willingness to participate when contacted by the researchers with more details, and 32 individuals formally took part by attending the first session. This result can be seen as an indication of the extent of recruitment required to meet target sample sizes. It is therefore not a conservative estimate to expect only $15 \%$ of those expressing initial willingness to participate in a MBI of that type to convert into an actual participant. Future studies may explore in more detail the barriers for participating as well as the characteristics of individuals who tend to express initial willingness to participate in contrast to those that eventually do participate.

\section{Limitations}


The participants were required to complete a number of questionnaires, which may have contributed to response fatigue. To limit this response burden, no measure of therapeutic alliance was included in the present study. However, for online interventions, issues of trust and alliance are likely to be equally important as for more traditional face-to-face formats, and more data on the challenges to building therapeutic alliance in online contexts will be necessary to understand the mechanism for treatment effectiveness and potential reason for non-adherence. Such research could explore the unique context of online environments for miscommunication and develop new strategies to address and avoid misunderstandings (Lee 2010). Even though the present study was not conducted in a therapeutic setting, the relationship between the course participants and the facilitator may still have been an important factor in the participants' motivations to attend sessions and conduct home practice.

The effect sizes reported here need to be interpreted in comparison with related programs that may be affected by the same kinds of self-selection biases that could have applied here. Reasons for practicing mindfulness can be very varied (Pepping et al. 2016), and it appears that intention to practice is related to the perceived benefits of meditation, rather than perceived severity of stressrelated problems (Rizer et al. 2016). Due to the low response rate and limited range of variability in scores, frequency and length of home practice could not be used in the present study as a co-variate. Additionally, the empirical evidence for the benefits of home practice is mixed (Lloyd et al. 2018; Ribeiro et al. 2018), and other ways of assessing practice, such as quality, may be necessary.

While the results highlight that the pilot videoconference-delivered mindfulness group program was effective in increasing mindfulness and producing significant positive changes on several relevant outcome measures, it is unclear how long-lasting these effects are likely to have been. Due to limited resources and the requirement to fit in with the schedule of concurrent studies on mindfulness and brain function (Wang et al. 2017), it was only possible to include one follow-up data collection point. This follow-up was conducted for only one of the groups and was conducted relatively soon, namely three weeks after the first post-intervention data collection. Effects from 
online therapy approaches may certainly decrease over time (Richard and Richardson 2012), and booster sessions may be beneficial. However, the present study highlighted that a videoconferencedelivered group format may provide a viable alternative if the main facilitator is located too far away to enable face-to-face contact. Certainly, the program still required the presence of locally based researchers to organize sessions, set up the equipment, and to be available to solve technical issues. It was not the intention to simulate a fully automated online intervention but only to explore to what extent the intervention may still work if the main facilitator is joining in part via videoconferencing. The results also indicate that having guest lecturers join via videoconferencing may not necessarily result in loss of effectiveness of MBIs. The online group format may also be useful for training purposes where the main facilitator and/or mentor may be located in another city or even country.

\section{Compliance with Ethical Standards}

All procedures performed in this study involving human participants were in accordance with the ethical standards of the institutional ethics committee of Auckland University of Technology, New Zealand, and with the 1964 Helsinki declaration and its later amendments or comparable ethical standards. Informed consent was obtained from all individual participants included in the study.

\section{References}

Abbott, R., Whear, R., Rodgers, L., Bethel, A., Thompson Coon, J., Kuyken, W.,..., \& Dickens, C. (2014). Effectiveness of mindfulness-based stress reduction and mindfulness based cognitive therapy in vascular disease: A systematic review and meta-analysis of randomised controlled trials. Journal of Psychosomatic Research, 76(5), 341-351. 
Aikens, K., Astin, J., Pelletier, K., Levanovich, K., Baase, C., Park, Y., \& Bodnar, C. (2014). Mindfulness goes to work: Impact of an online workplace intervention. Journal of Occupational and Environmental Medicine, 56(7), 721-731.

Andersson, G., \& Cuijpers, P. (2009). Internet-based and other computerized psychological treatments for adult depression: A meta-analysis. Cognitive Behaviour Therapy, 38(4), 196-205.

Arain, M., Campbell, M. J., Cooper, C. L., \& Lancaster, G. A. (2010). What is a pilot or feasibility study? A review of current practice and editorial policy. BMC Medical Research Methodology, 10:67.

Baer, R., Smith, G., Hopkins, J., Krietemeyer, J., \& Toney, L. (2006). Using self-report assessment methods to explore facets of mindfulness. Assessment, 13(1), 27-45.

Banerjee, M., Cavanagh, K., \& Strauss, C. (2018). Barriers to mindfulness: a path analytic model exploring the role of rumination and worry in predicting psychological and physical engagement in an online mindfulness-based intervention. Mindfulness, 9(3), 980-992.

Barak, A., Hen, L., Boniel-Nissim, M., \& Shapira, N. (2008). A comprehensive review and a metaanalysis of the effectiveness of internet-based psychotherapeutic interventions. Journal of Technology in Human Services, 26(2/4), 109-160.

Beck, A. T., Ward, C. H., Mendelssohn, M. J., \& Erbaugh, J. (1961). An inventory for measuring depression. Archives of General Psychiatry, 4, 561-571.

Beck, A. T., Epstein, N., Brown, G., \& Steer, R. A. (1988). An inventory for measuring clinical anxiety: Psychometric properties. Journal of Consulting and Clinical Psychology, 56, 893-897.

Beck, A. T., Steer, R. A., \& Brown, G. K. (1996). Beck Depression Inventory Manual, 2nd ed. San Antonio: The Psychological Corporation, Harcourt, Brace \& Company.

Beevers, C. G., Strong, D. R., Meyer, B., Pilkonis, P. A., \& Miller, I. W. (2007). Efficiently assessing negative cognition in depression: An Item Response Theory analysis of the Dysfunctional Attitude Scale. Psychological Assessment, 19(2), 199-209.

Brito-Pons, G., Campos, D., \& Cebolla, A. (2018). Implicit or explicit compassion? Effects of cultivation training and comparison with mindfulness-based stress reduction. Mindfulness. Online First. 
Broderick, P. C., Frank, J. L., Berrena, E., Schussler, D. L., Kohler, K., Mitra, J.,.., \& Greenberg, M. T. (2018). Evaluating the quality of mindfulness instruction delivered in school settings: development and validation of a teacher quality observational rating scale. Mindfulness. Online First.

Cavanagh, K., Strauss, C., Forder, L., \& Jones, F. (2014). Can mindfulness and acceptance be learnt by self-help?: A systematic review and meta-analysis of mindfulness and acceptance-based selfhelp interventions. Clinical Psychology Review, 34(2), 118-129.

Cavanagh, K., Churchard, A., O’Hanlon, P., Mundy, T., Votolato, P., Jones, F., Gu, J., \& Strauss, C. (2018). A randomised controlled trial of a brief online mindfulness-based intervention in a nonclinical population: Replication and extension. Mindfulness, 9(4), 1191-1205.

Christopher, M. S., Woodrich, L. E., \& Tiernan, K. A. (2014). Using cognitive interviews to assess the cultural validity of state and trait measures of mindfulness among Zen Buddhists. Mindfulness, 5(2), 145-160.

Devcich, D. A., Rix, G., Bernay, R., \& Graham, E. (2017). Effectiveness of a mindfulness-based program on school children's self-reported well-being: A pilot study comparing effects with an emotional literacy program. Journal of Applied School Psychology, 33(4), 309-330.

de Sá Junior, A. R., de Andrade, A. G., Andrade, L. H., Gorenstein, C., \& Wang, Y.-P. (2018).

Response patternof depressive symptoms among colleg students: What lies behind items of the Beck Depression Inventory-II?. Journal of Affective Disorders, 234, 124-130.

Ehring, T., Zetsche, U., Weidacker, K., Wahl, K., Schönfeld, S., \& Ehlers, A. (2011). The Perseverative Thinking Questionnaire (PTQ): Validation of a content-independent measure of repetitive negative thinking. Journal of Behavior Therapy and Experimental Psychiatry, 42(2), 225-232.

Feng, X. J., Krägeloh, C. U., Billington, D. R., \& Siegert, R. J. (2018). To what extent is mindfulness as presented in commonly used mindfulness questionnaires different from how it is conceptualized by senior ordained Buddhists?. Mindfulness, 9(2), 441-460. 
Fernandez, A. C., Wood, M. D., Stein, L. A. R., \& Rossi, J. S. (2010). Measuring mindfulness and examining its relationship with alcohol use and negative consequences. Psychology of Addictive Behaviors, 24(4), 608-616.

Fish, J., Brimson, J., \& Lynch, S. (2016). Mindfulness interventions delivered by technology without facilitator involvement: What research exists and what are the clinical outcomes?. Mindfulness, 7(5), 1011-1023.

Forbes, L., Gutierrez, D., \& Johnson, S. K. (2018). Investigating adherence to an online introductory mindfulness program. Mindfulness, 9(1), 271-282.

Gotink, R., Chu, P., Busschbach, J., Benson, H., Fricchione, G., \& Hunink, M. (2015). Standardised mindfulness-based interventions in healthcare: An overview of systematic reviews and metaanalyses of RCTs. PLoS ONE, 10(4), e0124344.

Gu, J., Cavanagh, K., \& Strauss, C. (2018). Investigating the specific effects of an online mindfulness-based self-help intervention on stress and underlying mechanisms. Mindfulness, $9(4), 1245-1257$.

Hayes, S., Strosahl, K., \& Wilson, K. (1999). Acceptance and commitment therapy: An experiential approach to behavior change. (1st ed.). New York: Guilford Press.

Henning, M. A., Krägeloh, C. U., Dryer, R., Moir, F., Billington, R., \& Hill, A. G. (Eds.) (2018). Wellbeing higher education: Cultivating a healthy lifestyle among faculty and students. Oxon, United Kingdom: Routledge.

Julian, L. J. (2011). Measures of anxiety - State-Trait Anxiety Inventory (STAI), Beck Anxiety Inventory (BAI), and Hospital Anxiety and Depression Scale-Anxiety (HADS-A). Arthritis Care \& Research, 63(S11), S467-S472.

Kabat-Zinn, J. (1990). Full catastrophe living. New York, NY: Delacourt.

Khoury, B., Sharma, M., Rush, S. E., \& Fournier, C. (2015). Mindfulness-based stress reduction for healthy individuals: a meta-analysis. Journal of Psychosomatic Research, 78(6), 519-528.

Kirk, A. (2013). The effect of newer communication technologies on relationship maintenance and satisfaction in long-distance dating relationships. Pepperdine Journal of Communication Research, 1(2), 3-7. 
Krägeloh, C. U. (2016). Importance of morality in mindfulness practice. Counseling and Values, 61(1), 97-110.

Lacaille, J., Sadikaj, G., Nishioka, M., Carrière, K., Flanders, J., \& Knäuper, B. (2017). Daily mindful responding mediates the effect of meditation practice on stress and mood: The role of practice duration and adherence. Journal of Clinical Psychology, 74(1), 109-122.

Lauricella, S. (2014). Mindfulness meditation with undergraduates in face-to-face and digital practice: a formative analysis. Mindfulness, 5(6), 682-688.

Lee, S. (2010). Frontline perspectives: Contemporary issues of ethical e-therapy. Journal of Ethics in Mental Health, 5(1), 1-5.

Lloyd, A., White, R., Eames, C., \& Crane, R. (2018). The utility of home-practice in mindfulnessbased group interventions: A systematic review. Mindfulness, 9(3), 673-692.

Magtibay, D. L., Chesak, S. S., Coughlin, K., \& Sood, A. (2017). Decreasing stress and burnout in nurses - efficacy of blended learning with stress management and resilience training program. Journal of Nursing Administration, 47(7/8), 391-395.

Medvedev, O. N., Siegert, R. J., Kersten, P., \& Krägeloh, C. U. (2017). Improving the precision of the Five Facet Mindfulness Questionnaire using a Rasch approach. Mindfulness, 8(4), 995-1008.

Montero-Marin, J., Gaete, J., Araya, R., Demarzo, M., Manzanera, R., Álvarez de Mon, M., \& García-Campayo, J. (2018). Impact of a blended web-based mindfulness programme for general practitioners: a pilot study. Mindfulness, 9(1), 129-139.

Neff, K. D. (2003). The development and validation of a scale to measure self-compassion. Self and Identity, 2(3), 223-250.

Pepping, C. A., Walters, B., Davis, P. J., \& O’Donovan, A. (2016). Why do people practice mindfulness? An investigation into reasons for practicing mindfulness meditation. Mindfulness, $7(2), 542-547$.

Plaza, I., Demarzo, M. M. P., Herrera-Mercadal, P., \& García-Campayo, J. (2013). Mindfulness-based mobile applications: Literature review and analysis of current features. JMIR mHealth and uHealth, 1(2), e24.

Pommier, E. A. (2010). The compassion scale. PhD dissertation. Retrieved from 
https://repositories.lib.utexas.edu/handle/2152/ETD-UT-2010-12-2213.

Raes, F., Pommier, E., Neff, K. D., \& Van Gucht, D. (2011). Construction and factorial validation of a short form of the self-compassion scale. Clinical Psychology and Psychotherapy, 18(3), 250255.

Ribeiro, L., Atchley, R. M., \& Oken, B. S. (2018). Adherence to practice of mindfulness in novice meditators: practices chosen, amount of time practiced, and long-term effects following a mindfulness-based intervention. Mindfulness, 9(2), 401-411.

Richards, D., \& Richardson, T. (2012). Computer-based psychological treatments for depression: a systematic review and meta-analysis. Clinical Psychology Review, 32(4), 329-342.

Rizer, C. A., Fagan, M. H., Kilmon, C., \& Rath, L. (2016). The role of perceived stress and health beliefs on college students' intentions to practice mindfulness meditation. American Journal of Health Education, 47(1), 24-31.

Rudkin, E., Medvedev, O. N., Siegert, R. J. (2018). The Five-Facet Mindfulness Questionnaire: Why the Observing facet does not predict psychological symptoms. Mindfulness, 9(1), 230-242.

Salmoirago-Blotcher, E., Carmody, J., Yeh, G., Crawford, S., Rosenthal, L., \& Ockene, I. (2012). Design and methods for a pilot study of a phone-delivered, mindfulness-based intervention in patients with implantable cardioverter defibrillators. Evidence-Based Complementary and Alternative Medicine, 12, Article ID 972106.

Siegel, S., \& Castellan, N. J. (1988). Nonparametric statistics for the behavioral sciences. New York, NY: McGraw-Hill.

Siegert, R. J., Walkey, F. H., \& Turner-Stokes, L. (2009). An examination of the factor structure of the Beck Depression Inventory-II in a neurorehabilitation inpatient sample. Journal of the International Neuropsychological Society, 15(1), 142-147.

Spijkerman, M., Pots, W., \& Bohlmeijer, E. (2016). Effectiveness of online mindfulness-based interventions in improving mental health: A review and meta-analysis of randomised controlled trials. Clinical Psychology Review, 45, 102-114. 
Tennant, A., \& Conaghan, P. G. (2007). The Rasch measurement model in rheumatology: what is it and why use it? When should it be applied, and what should one look for in a Rasch paper?. Arthritis \& Rheumatism, 57(8), 1358-1362.

Thabane, L., Ma, J., Rong, C., Cheng, J., Ismaila, A., Rios, L. P.,..., \& Goldsmith, C. H. (2010). A tutorial on pilot studies: the what, why and how. BMC Medical Research Methodology, 10:1.

Thompson, M., \& Walter, F. (2016). Increases in general practice workload in England. Lancet, 387(10035), 2270-2272.

Tsaousides, T., D’Antonio, E., Varbanova, V., \& Spielman, L. (2014). Delivering group treatment via videoconference to individuals with traumatic brain injury: A feasibility study.

Neuropsychological Rehabilitation, 24(5), 784-803.

van Emmerick, A. A. P., Berings, F., \& Lancee, J. (2018). Efficacy of a mindfulness-based mobile application: a randomized waiting-list controlled trial. Mindfulness, 9(1), 187-198.

Wang, G. Y., Taylor, T., Sumich, A., Merien, F., Borotkanics, R., Wrapson, W.,..., \& Siegert, R. J. (2017). Associations between immunological function and memory recall in healthy adults. Brain \& Cognition, 119, 39-44.

Watson, D., Clark, L. A., \& Tellegen, A. (1988). Development and validation of brief measures of positive and negative affect: The PANAS scales. Journal of Personality and Social Psychology, 54(6), 1063-1070.

Williams, J., Teasdale, J., Segal, Z., \& Kabat-Zinn, J. (2007). The mindful way through depression (1st ed.). New York: Guilford Publications. 


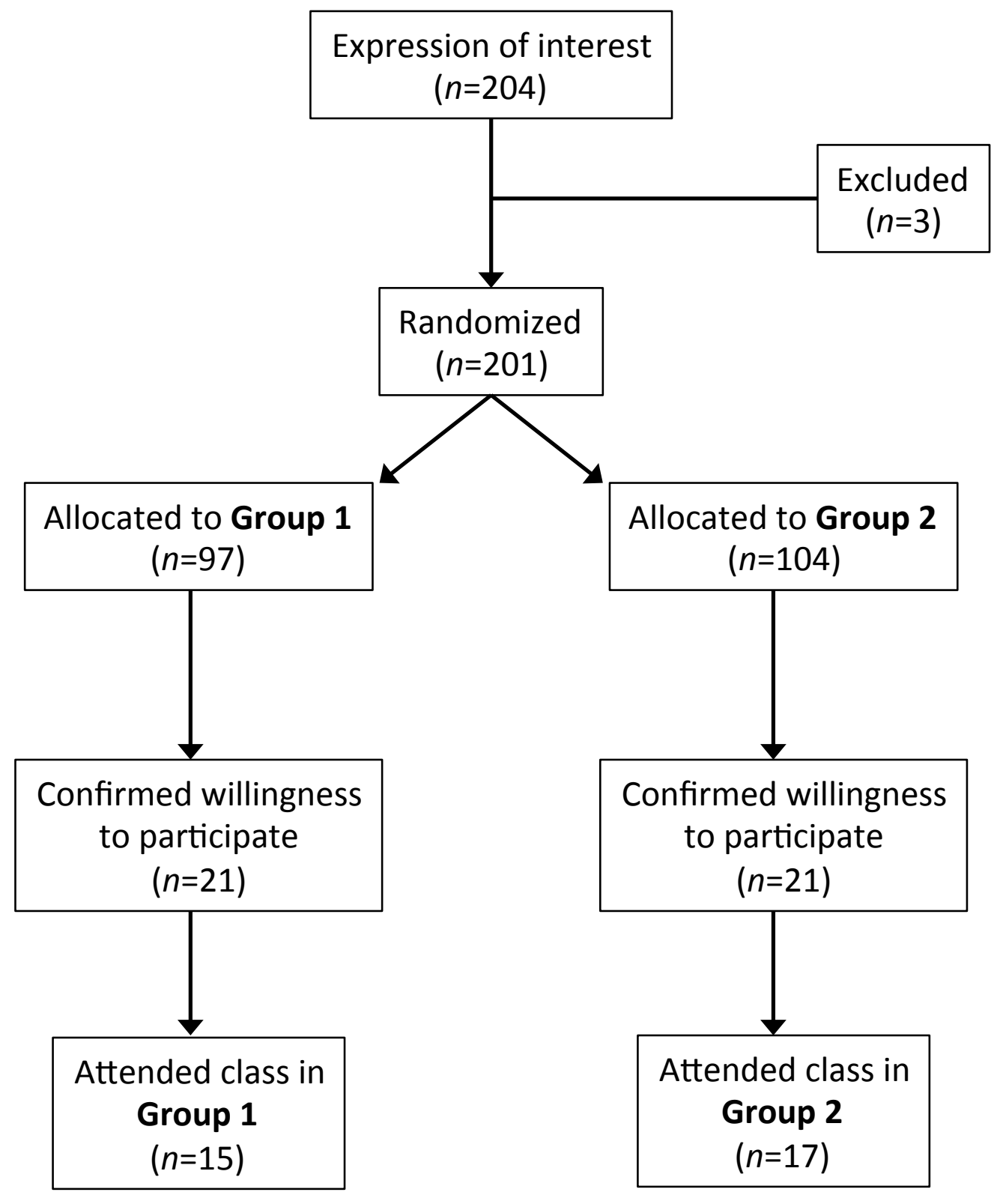


Table 1 Session-by-session outline of the videoconference-delivered mindfulness-based group program.

\begin{tabular}{|c|c|}
\hline $\begin{array}{l}\text { Session } 1 \\
\text { (face to face) }\end{array}$ & $\begin{array}{l}\text { "Already here, always now" } \\
\text { Introductory ice-breaker exercise on wellbeing } \\
\text { Facilitator introduced himself and spoke about his personal mindfulness practice } \\
\text { Guided meditation focusing on breathing }\end{array}$ \\
\hline Session 2 & $\begin{array}{l}\text { "Shifting the auto-pilot" } \\
\text { Guided breathing meditation } \\
\text { Mindful tasting } \\
\text { Powerpoint presentation and discussion: mindfulness and neuroscience } \\
\text { Physical exercises with focus on breathing } \\
\text { Guided breathing meditation }\end{array}$ \\
\hline Session 3 & $\begin{array}{l}\text { "Alright right now" } \\
\text { Physical exercises with focus on breathing } \\
\text { Mindfulness meditation } \\
\text { Guided breathing exercise } \\
\text { Body scan } \\
\text { Powerpoint presentation and discussion: negativity bias, types of awareness, } \\
\text { narratives when learning to meditate, walking meditation } \\
\text { Loving kindness meditation } \\
\text { Discussion }\end{array}$ \\
\hline Session 4 & $\begin{array}{l}\text { "Making space" } \\
\text { Concentration meditation focusing on breathing } \\
\text { Powerpoint presentation and discussion: four foundations of mindfulness, urge } \\
\text { surfing, transience of emotions, throwing out your anchor } \\
\text { Physical exercises with focus on breathing } \\
\text { Guided meditation with instructions to observe sounds, body, and emotions } \\
\text { Questions and answers }\end{array}$ \\
\hline Session 5 & $\begin{array}{l}\text { "Awareness, pure and simple" } \\
\text { Concentration meditation focusing on breathing } \\
\text { Powerpoint presentation and discussion: mindfulness and dealing with emotions } \\
\text { Physical exercises with focus on breathing } \\
\text { Concentration meditation focusing on breathing } \\
\text { Questions and answers }\end{array}$ \\
\hline Session 6 & $\begin{array}{l}\text { "Heart in mind" } \\
\text { Physical exercises with focus on breathing } \\
\text { Concentration meditation } \\
\text { Powerpoint presentation and discussion: more on four foundations of mindfulness, } \\
\text { mindfulness of mind objects, loving kindness meditation } \\
\text { Questions and answers } \\
\text { Feedback and discussion }\end{array}$ \\
\hline
\end{tabular}


Table 2 Overview of experimental timeline and measurement points. Pre1 refers to the baseline measure taken immediately prior to the interventions in Groups 1 and 2, while Pre0 refers to the first of the two baseline measures taken for Group 2. Post1 is the first post-intervention measure immediately after the intervention for Groups 1 and 2, and Post2 refers to the follow-up measure for Group 1.

\begin{tabular}{lll}
\hline Week & \multicolumn{1}{c}{ Group 1 } & \multicolumn{1}{c}{ Group 2 } \\
\hline 1 & Pre1 baseline measure $(n=13)$ & Pre0 baseline measure $(n=17)$ \\
2 & $1^{\text {st }}$ week of mindfulness program & \\
3 & $2^{\text {nd }}$ week of mindfulness program & Pre1 baseline measure $(n=16)$ \\
4 & $3^{\text {rd }}$ week of mindfulness program & $1^{\text {st }}$ week of mindfulness program \\
5 & $4^{\text {th }}$ week of mindfulness program & $2^{\text {nd }}$ week of mindfulness program \\
6 & $5^{\text {th }}$ week of mindfulness program & $3^{\text {rd }}$ week of mindfulness program \\
7 & $6^{\text {th }}$ week of mindfulness program & $4^{\text {th }}$ week of mindfulness program \\
8 & Post1 post-intervention measure $(n=12)$ & $5^{\text {th }}$ week of mindfulness program \\
9 & & $6^{\text {th }}$ week of mindfulness program \\
10 & & Post1 post-intervention measure $(n=11)$ \\
11 & Post2 post-intervention measure $(n=11)^{*}$ & Post
\end{tabular}

${ }^{\star}$ At Post2 for Group 1, only 10 participants completed the DAS.

Table 3 Course attendance by session ( $n$ and \%), shown separately for Groups 1 and 2.

\begin{tabular}{lcccccc}
\hline & Session & Session & Session & Session & Session & Session \\
& 1 & 2 & 3 & 4 & 5 & 6 \\
\hline Group 1 $(n=15)$ & 15 & $14(93 \%)$ & $12(80 \%)$ & $6(40 \%)$ & $7(47 \%)$ & $10(67 \%)$ \\
& $(100 \%)$ & & & & & \\
Group 2 $(n=17)$ & $15(88 \%)$ & $15(88 \%)$ & $12(71 \%)$ & $9(53 \%)$ & $9(53 \%)$ & $13(76 \%)$
\end{tabular}


Table 4 Means (M) and standard deviations (SD) for each of the outcomes measures, presented separately for the full sample of participants with Pre1 and Post1 scores $(n=22)$, those who only attended three or four sessions of the mindfulness program ( $n=7)$ and those who attended five or six sessions ( $n=15)$. Cohen's $d$ effect sizes refer to Pre1-to-Post1 comparisons.

\begin{tabular}{|c|c|c|c|c|c|c|c|c|c|}
\hline \multirow[t]{2}{*}{ Outcome Measure } & \multirow{2}{*}{$\begin{array}{l}\begin{array}{l}\text { Pre1 all } \\
(n=22)\end{array} \\
\mathrm{M}(\mathrm{SD})\end{array}$} & \multicolumn{2}{|c|}{ Post1 all $(n=22)$} & \multirow{2}{*}{$\begin{array}{l}\text { Pre1 for } 3 \text { or } 4 \\
\text { sess. }(n=7) \\
\text { M (SD) }\end{array}$} & \multicolumn{2}{|c|}{$\begin{array}{l}\text { Post } 1 \text { for } 3 \text { or } 4 \\
\text { sess. }(n=7)\end{array}$} & \multirow{2}{*}{$\begin{array}{l}\text { Pre1 for } 5 \text { or } 6 \text { sess. } \\
(n=15) \\
M(S D)\end{array}$} & \multicolumn{2}{|c|}{$\begin{array}{l}\text { Post1 for } 5 \text { or } 6 \\
\text { sess. }(n=15)\end{array}$} \\
\hline & & $M(S D)$ & $d$ & & $M(S D)$ & $d$ & & M (SD) & $d$ \\
\hline BDI & $\begin{array}{l}11.64 \\
(9.46)\end{array}$ & $\begin{array}{l}6.00 \\
(4.93)\end{array}$ & $-0.75^{\star \star}$ & $7.14(4.88)$ & $\begin{array}{l}4.71 \\
(4.96)\end{array}$ & -0.49 & $13.73(10.46)$ & $\begin{array}{l}6.60 \\
(4.97)\end{array}$ & $-0.87^{\star *}$ \\
\hline BAI & $\begin{array}{l}8.59 \\
(6.77)\end{array}$ & $\begin{array}{l}7.55 \\
(7.01)\end{array}$ & -0.15 & $5.57(2.99)$ & $\begin{array}{l}7.71 \\
(9.29)\end{array}$ & 0.31 & $10.00(7.63)$ & $\begin{array}{l}7.47 \\
(6.06)\end{array}$ & $-0.37^{*}$ \\
\hline DAS & $\begin{array}{l}24.32 \\
(5.12)\end{array}$ & $\begin{array}{l}26.00 \\
(3.92)\end{array}$ & $0.37^{*}$ & $25.00(2.77)$ & $\begin{array}{l}26.00 \\
(2.83)\end{array}$ & 0.36 & $24.00(5.98)$ & $\begin{array}{l}26.00 \\
(4.47)\end{array}$ & $0.38^{*}$ \\
\hline PTQ & $\begin{array}{l}31.77 \\
(14.40)\end{array}$ & $\begin{array}{l}26.41 \\
(8.20)\end{array}$ & $-0.46^{*}$ & $28.86(15.36)$ & $\begin{array}{l}23.86 \\
(5.58)\end{array}$ & -0.43 & $33.13(14.27)$ & $\begin{array}{l}27.60 \\
(9.09)\end{array}$ & $-0.46^{*}$ \\
\hline PA & $\begin{array}{l}33.59 \\
(8.80)\end{array}$ & $\begin{array}{l}33.45 \\
(8.77)\end{array}$ & -0.02 & $37.43(7.28)$ & $\begin{array}{l}33.14 \\
(8.36)\end{array}$ & -0.55 & $31.80(9.09)$ & $\begin{array}{l}33.60 \\
(9.23)\end{array}$ & 0.20 \\
\hline NA & $\begin{array}{l}22.86 \\
(8.43)\end{array}$ & $\begin{array}{l}18.64 \\
(5.64)\end{array}$ & $-0.59^{*}$ & $21.14(6.31)$ & $\begin{array}{l}19.00 \\
(6.30)\end{array}$ & -0.34 & $23.67(9.34)$ & $\begin{array}{l}18.47 \\
(5.53)\end{array}$ & $-0.68^{*}$ \\
\hline SCS & $\begin{array}{l}31.18 \\
(9.45)\end{array}$ & $\begin{array}{l}37.41 \\
(7.70)\end{array}$ & $0.72^{\star \star}$ & $34.71(7.46)$ & $\begin{array}{l}37.57 \\
(4.83)\end{array}$ & 0.46 & $29.53(10.04)$ & $\begin{array}{l}37.33 \\
(8.88)\end{array}$ & $0.82^{* *}$ \\
\hline CS & $\begin{array}{l}94.82 \\
(11.24)\end{array}$ & $\begin{array}{l}95.95 \\
(14.20)\end{array}$ & 0.09 & $87.71(13.05)$ & $\begin{array}{l}82.57 \\
(12.25)\end{array}$ & -0.41 & $98.13(8.91)$ & $\begin{array}{l}102.20 \\
(10.34)\end{array}$ & 0.42 \\
\hline FFMQ total & $\begin{array}{l}112.78 \\
(7.80)\end{array}$ & $\begin{array}{l}116.71 \\
(5.75)\end{array}$ & $0.57^{* *}$ & $113.55(7.74)$ & $\begin{array}{l}113.47 \\
(2.64)\end{array}$ & -0.01 & $112.43(8.07)$ & $\begin{array}{l}118.22 \\
(6.25)\end{array}$ & $0.80^{\star *}$ \\
\hline FFMQ Act & $\begin{array}{l}24.58 \\
(3.23)\end{array}$ & $\begin{array}{l}26.15 \\
(3.06)\end{array}$ & 0.50 & $25.04(1.95)$ & $\begin{array}{l}24.85 \\
(3.21)\end{array}$ & -0.07 & $24.36(3.73)$ & $\begin{array}{l}26.75 \\
(2.91)\end{array}$ & $0.71^{\star *}$ \\
\hline FFMQ Describe & $\begin{array}{l}21.14 \\
(5.23)\end{array}$ & $\begin{array}{l}22.08 \\
(3.36)\end{array}$ & 0.21 & $22.71(3.03)$ & $\begin{array}{l}22.38 \\
(2.80) \\
\end{array}$ & -0.11 & $20.41(5.95)$ & $\begin{array}{l}21.95 \\
(3.67) \\
\end{array}$ & 0.31 \\
\hline FFMQ Nonjudge & $\begin{array}{l}25.12 \\
(5.27) \\
\end{array}$ & $\begin{array}{l}27.56 \\
(5.30)\end{array}$ & $0.46^{*}$ & $25.28(7.22)$ & $\begin{array}{l}25.78 \\
(2.89) \\
\end{array}$ & 0.09 & $25.05(4.39)$ & $\begin{array}{l}28.39 \\
(6.02) \\
\end{array}$ & $0.63^{*}$ \\
\hline FFMQ Nonreact & $\begin{array}{l}16.60 \\
(3.29)\end{array}$ & $\begin{array}{l}17.27 \\
(2.47)\end{array}$ & 0.23 & $17.90(3.16)$ & $\begin{array}{l}17.51 \\
(2.23)\end{array}$ & -0.14 & 15.99 (3.27) & $\begin{array}{l}17.16 \\
(2.64)\end{array}$ & $0.39^{*}$ \\
\hline FFMQ Observe & $\begin{array}{l}24.64 \\
(2.90) \\
\end{array}$ & $\begin{array}{l}25.34 \\
(2.84) \\
\end{array}$ & 0.24 & $23.20(3.17)$ & $\begin{array}{l}23.04 \\
(3.30)\end{array}$ & -0.05 & $25.31(2.60)$ & $\begin{array}{l}26.41 \\
(1.87)\end{array}$ & 0.49 \\
\hline
\end{tabular}

${ }^{*} p<.05,{ }^{* *} p<.01$ 